\title{
Automaticity in Cardiomyocytes Derived From Human Induced Pluripotent Stem Cells as Result of Different Mechanisms
}

\author{
Michelangelo Paci ${ }^{1}$, Stefano Severi ${ }^{2}$, Jari Hyttinen ${ }^{1}$ \\ ${ }^{1}$ BioMediTech, Faculty of Medicine and Health Technology, Tampere University, Tampere, Finland \\ ${ }^{2}$ Department of Electrical, Electronic and Information Engineering, University of Bologna, Cesena, \\ Italy
}

\begin{abstract}
Cardiomyocytes derived from human induced pluripotent stem cells (hiPSC-CMs) are nowadays one of the most studied cell types, especially for their role as in vitro models to test drug safety. Here we propose an update of our recent Paci2018 hiPSC-CM model, focusing particularly on the mechanisms underlying the action potential $(A P)$ automaticity, i.e. one of the clearest markers of hiPSC-CM immaturity. We used two recently published formulations of the fast $\mathrm{Na}^{+}\left(I_{\mathrm{Na}}\right)$ and the funny $\left(I_{f}\right)$ currents to improve our previous fitting of in vitro experiments. We then used an optimization framework to automatically identify the parameters formalizing currents/mechanisms for which in vitro data were not available. We identified a model producing APs and $\mathrm{Ca}^{2+}$ transients (CaTrs) in agreement with literature and our in-house CaTr data. We showed that a strong (90\%) $\mathrm{Na}^{+} / \mathrm{Ca}^{2+}$ exchanger ( $\left.\mathrm{I}_{\mathrm{NCX}}\right)$ block suppresses the spontaneous APs, in agreement with in vitro data. The new Paci2019 was further validated against the in vitro experiments used for the Paci2018 model, showing that this update did not affect the capability of the new model to simulate mechanisms (e.g. responses to drugs or proarrhythmic events) successfully reproduced by the previous model. In conclusion, the Paci2019 model represents an improved tools for in silico studies e.g. on hiPSC-CM responses to drugs.
\end{abstract}

\section{Introduction}

Cardiomyocytes derived from human induced pluripotent stem cells (hiPSC-CMs) are nowadays extensively studied, especially for their role as in vitro models to test drug safety. In the last decade, the amount of available in vitro data increased considerably, together with our understanding of hiPSC-CM electrophysiology. Experiments supported hiPSC-CM use as drug prescreening models (e.g. in the Comprehensive in Vitro Proarrhythmia Assay initiative - CiPA), but also partly dampened the initial enthusiasm because of hiPSC-CM structural immaturity. Together with the increasing amount of in vitro data, more and more in silico studies helped to shed light on hiPSC-CM electrophysiology and responses to drugs [1-6].

Two mechanisms are currently considered responsible for automaticity in hiPSC-CMs: i) membrane potential depolarized by the funny current $\left(\mathrm{I}_{\mathrm{f}}\right)$ [7] or ii) by the $\mathrm{Na}^{+} / \mathrm{Ca}^{2+}$ exchanger $\left(\mathrm{I}_{\mathrm{NCX}}\right)$ as consequence of $\mathrm{Ca}^{2+}$ release from sarcoplasmic reticulum (SR) [8]. Currently, there is no consensus, also because of contradicting in vitro experiments.

We observed a limitation in our recent Paci2018 hiPSC-CM model [3], i.e. a too strong window current for the fast $\mathrm{Na}^{+}$current $\left(\mathrm{I}_{\mathrm{Na}}\right)$, which makes $\mathrm{I}_{\mathrm{Na}}$ not only fundamental for the upstroke phase of the AP, but also in sustaining the automaticity of the spontaneous electrical activity. Because of this, the Paci2018 model failed to simulate the cessation of the spontaneous electrical activity in case of strong block of $\mathrm{Na}^{+} / \mathrm{Ca}^{2+}$ exchanger ( $\left.\mathrm{I}_{\mathrm{NCX}}\right)$ as shown by recent in vitro and in silico experiments $[4,8]$.

Here we propose an update of our Paci2018 hiPSC-CM model [3], focusing particularly on the mechanisms underlying the action potential (AP) automaticity, i.e. one of the clearest markers of hiPSC-CM immaturity.

\section{Methods}

In order to improve the simulation of the spontaneous hiPSC-CM electrical activity we proceeded in three steps:

- replacement of $\mathrm{I}_{\mathrm{Na}}$ and funny current $\left(\mathrm{I}_{\mathrm{f}}\right)$ formulation and other minor changes;

- $\quad$ automatic parameter optimization;

- validation of the model against the in vitro experiments used to validate the Paci2018 model.

\subsection{New ion current formulations}

We first replaced the formulation of $\mathrm{I}_{\mathrm{Na}}$ and of $\mathrm{I}_{\mathrm{f}}$, with new ones based on [4], that provided an improved fitting of the in vitro voltage-clamp experiments from $[9,10]$. 
Since these changes suppressed the model automaticity, we manually tuned: the maximum $\mathrm{Na}^{+} / \mathrm{Ca}^{2+}$ exchanger $\left(\mathrm{I}_{\mathrm{NCX}, \text { max }}\right)$, the $\mathrm{Na}^{+} / \mathrm{K}^{+}$pump $\left(\mathrm{I}_{\mathrm{NaK}, \text { max }}\right)$, the maximum $\mathrm{Na}^{+}$ and $\mathrm{Ca}^{2+}$ background current conductances $\left(\mathrm{G}_{\mathrm{b}, \mathrm{Na}}\right.$ and $\left.\mathrm{G}_{\mathrm{b}, \mathrm{Ca}}\right)$ and the maximum SERCA uptake $\left(\mathrm{V}_{\text {max,up }}\right)$. This led to a model showing spontaneous electrical activity and biomarkers similar to those reported in [3].

\subsection{Automatic parameter optimization}

Since this intermediate model did not simulate all the AP and CaTr biomarkers simulated by the Paci2018 model within their in vitro ranges, we then run the same automatic optimization algorithm we used in [3]. The target AP in vitro biomarkers, taken from [9] and considered for the cost function, were: AP amplitude (APA), maximum diastolic potential (MDP), cycle length (CL), maximum upstroke velocity $\left(\mathrm{dV} / \mathrm{dt}_{\max }\right), \mathrm{AP}$ duration at 10,30 and $90 \%$ of repolarization $\left(\mathrm{APD}_{10}, \mathrm{APD}_{30}, \mathrm{APD}_{90}\right)$ and $\mathrm{AP}$ shape factor (Tri), i.e. the ratio $\left(A P D_{30}-A P D_{40}\right) /$ $\left(A P D_{70}-A P D_{80}\right)$. The target $\mathrm{Ca}^{2+}$ transients (CaTrs) in vitro biomarkers, used for the Paci2018 model and recorded at BioMediTech (Tampere, Finland) [3], were: CaTr duration (CTD), time to peak (tRise 10 ,peak), rise time from $10 \%$ to $50 \%$ (tRise 10,50 ), rise time from $10 \%$ to $90 \%$ (tRise ${ }_{10,90}$ ), decay time from $90 \%$ to $10 \%$ (tDecay90,10) and the CaTr cycle length (CaTr CL). We optimized the same parameters as in [3]. The parameter values were constrained in a range $[-20 \%,+20 \%]$ with respect to their nominal value in the Paci2018 model, in order to avoid non-physiological values.

\subsection{Model validation}

To validate the new hiPSC-CM model we considered the following data:

- administration of SEA0400 ( $\mathrm{I}_{\mathrm{NCX}}$ blocker) and ivabradine ( $\mathrm{I}_{\mathrm{f}}$ blocker) as in [8];

- $\quad$ spontaneous $\mathrm{Ca}^{2+}$ release from $\mathrm{SR}$ in case of $\mathrm{Ca}^{2+}$ overload [8] and due to abnormal $\mathrm{Ca}^{2+}$ handling;

- administration of tetrodotoxine ( $\mathrm{I}_{\mathrm{Na}}$ blocker), nifedipine $\left(\mathrm{I}_{\mathrm{CaL}}\right.$ blocker), E4031 ( $\mathrm{I}_{\mathrm{Kr}}$ blocker) and 3R4S-Chromanol 293B ( $\mathrm{I}_{\mathrm{Ks}}$ blocker) [9];

\section{Results}

\subsection{Automaticity in the Paci2019 model}

The replacement of $\mathrm{I}_{\mathrm{Na}}$ and $\mathrm{I}_{\mathrm{f}}$ and the parameter optimization produced the APs and CaTrs shown in Fig.1, whose biomarkers are reported in Table 1. Furthermore, in spite our $\mathrm{CaTr}$ biomarkers did not include absolute measurements of CaTr amplitude, in [11] they observed CaTr amplitude ranging from 30 to $160 \mathrm{nM}$ in hiPSC-CM ensembles incubated at $37^{\circ} \mathrm{C}$ (calibrated Fura-2-based photometry measures). Our model produces CaTrs with amplitude 160nM.

The main difference in terms of ion currents between the Paci2018 and the Paci2019 models is the $\mathrm{I}_{\mathrm{NCX}}$ shape (Fig. 1). In fact, by reducing the $\mathrm{I}_{\mathrm{Na}}$ window current and using a more accurate fitting of $\mathrm{I}_{\mathrm{f}}$, we unveiled a remarkable inward $\mathrm{I}_{\mathrm{NCX}}$ inward component right before the AP upstroke, which significantly contributes to the membrane potential depolarization. This is indeed caused by the $\mathrm{Ca}^{2+}$ release from the sarcoplasmic reticulum (SR), which now assumes an important role in the model spontaneous electrical activity. Indeed, increasing blocks of $\mathrm{I}_{\mathrm{NCX}}$ first slow down the rate of the spontaneous APs, until their suppression (Fig. 2), as observed in vitro as consequence of the $\mathrm{I}_{\mathrm{NCX}}$ inhibitor SEA0400 [8]. As in $[7,8]$, we tested also the effect of $I_{\mathrm{f}}$ block. The effect of a $41 \%$ block, corresponding to $3 \mu \mathrm{M}$ ivabradine [4], induced only a slight reduction of AP rate $(-7.5 \%)$ in agreement with [8]. However, for increasing blocks, the effect on AP rate was more relevant: $60 \% \mathrm{I}_{\mathrm{f}}$ block caused $-12 \%$ AP rate and $80 \%$ block induced the cessation of the spontaneous APs after 260s from drug administration, in line with [7].

Table 1. AP and CaTr biomarkers: in vitro intervals used for parameter optimization and in silico values.

\begin{tabular}{|c|c|c|}
\hline Biomarker & $\begin{array}{l}\text { In vitro value } \\
(\text { mean } \pm \mathrm{SD})\end{array}$ & $\begin{array}{l}\text { In silico } \\
\text { value }\end{array}$ \\
\hline APA $(\mathrm{mV})$ [9] & $104 \pm 6.0$ & 102.0 \\
\hline $\operatorname{MDP}(\mathrm{mV})$ [9] & $-75.6 \pm 6.6$ & -74.9 \\
\hline AP CL (ms) [9] & $1700.0 \pm 547.7$ & 1712.4 \\
\hline $\mathrm{dV} / \mathrm{dt}_{\max }(\mathrm{V} / \mathrm{s})[9]$ & $27.8 \pm 26.3$ & 20.4 \\
\hline $\mathrm{APD}_{10}(\mathrm{~ms})[9]$ & $74.1 \pm 26.3$ & 87.0 \\
\hline $\mathrm{APD}_{30}(\mathrm{~ms})[9]$ & $180 \pm 58.6$ & 223.8 \\
\hline $\mathrm{APD}_{90}(\mathrm{~ms})$ [9] & $414.7 \pm 119.4$ & 390.2 \\
\hline AP Tri (-)[9] & $2.5 \pm 1.1$ & 2.8 \\
\hline CTD (ms) [3] & $804.5 \pm 188.0$ & 691.5 \\
\hline $\mathrm{CaTr} \operatorname{Rise}_{10,50}(\mathrm{~ms})$ [3] & $82.9 \pm 50.5$ & 55.0 \\
\hline $\mathrm{CaTr} \operatorname{Rise}_{10,90}(\mathrm{~ms})$ [3] & $167.3 \pm 69.8$ & 118.2 \\
\hline $\mathrm{CaTr}_{\text {tRise }}{ }_{10, \text { peak }}(\mathrm{ms})[3]$ & $270.4 \pm 108.3$ & 184.0 \\
\hline CaTr tDecay $_{90,10}(\mathrm{~ms})[3]$ & $409.8 \pm 100.1$ & 341.0 \\
\hline $\mathrm{CaTr}$ CL (ms) [3] & $1653.9 \pm 630$ & 1712.4 \\
\hline
\end{tabular}

\subsection{Delayed After-depolarizations}

As for the Paci2018 model, we tested the Paci2019 capability of producing delayed after-depolarizations (DADs) in different conditions. We induced $\mathrm{Ca}^{2+}$ overload, by increasing the extracellular $\mathrm{Ca}^{2+}$ concentration (2.9 from $1.8 \mathrm{mM}$ ) [12]. Fig. 3 shows how $\mathrm{Ca}^{2+}$ overload triggers spontaneous releases from SR that starts DADs (grey arrows) or even single and multiple anticipated APs. A similar result was obtained with a normal extracellular 

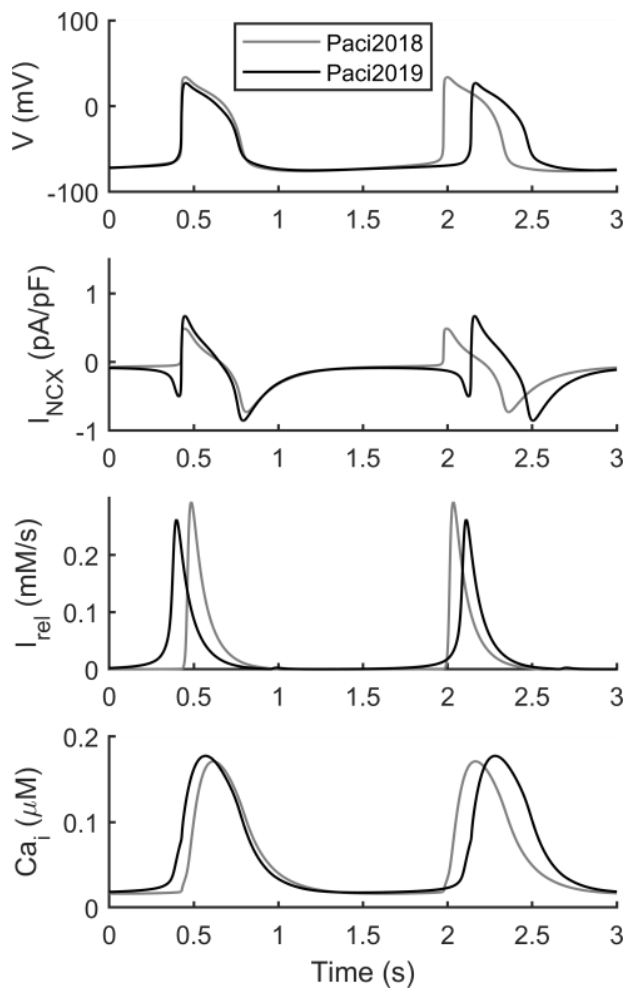

Figure 1. Paci2019 model. From top to bottom: APs, $\mathrm{I}_{\mathrm{NCX}}$, $\mathrm{I}_{\mathrm{rel}}$ and CaTrs.

$\mathrm{Ca}^{2+}$ concentration $(1.8 \mathrm{mM})$ but simulating an abnormal RyR machinery (namely, by shifting $\mathrm{RyR}_{\mathrm{o}, \text { half }}$ and $\mathrm{RyR}_{\mathrm{c}, \text { half }}$ by -0.002 and $0.002 \mathrm{mM}$ respectively, doubling $\mathrm{RyR}_{\mathrm{o}}$ time constant and reducing to half of its nominal value $\mathrm{RyR}_{\mathrm{c}}$ time constant) (Fig. 4).

\subsection{Response to current blockers}

We tested the Paci2019 model with four current blockers tested in vitro in [9]: tetrodotoxin, E4031, nifedipine and 3R4S-Chromanol 293B. As the in vitro hiPSC-CM, the model was stimulated at a constant pacing rate $1 \mathrm{~Hz}$ with depolarizing pulses of $5 \mathrm{~ms}$ duration. In agreement with the in vitro experiments (i) tetrodotoxine delays the upstroke, (ii) E4031 prolongs APD, (iii) nifedipine shortens APD and (iv) 3R4S-Chromanol 293B has only minor effects on the AP shape (Fig. 5).

\section{Conclusions}

Automaticity is one of the main hiPSC-CM features and there is no consensus on its triggering mechanism $\left(\mathrm{I}_{\mathrm{f}} \mathrm{vs}\right.$ $\mathrm{I}_{\mathrm{NCX}}+\mathrm{Ca}^{2+}$ releases). A reasonable hypothesis is that both mechanisms could coexist and the in vitro data supporting one or the other could be explained by the phenotypical variability (together with different in vitro setups, e.g. single cell data vs cell ensemble recordings). In this work
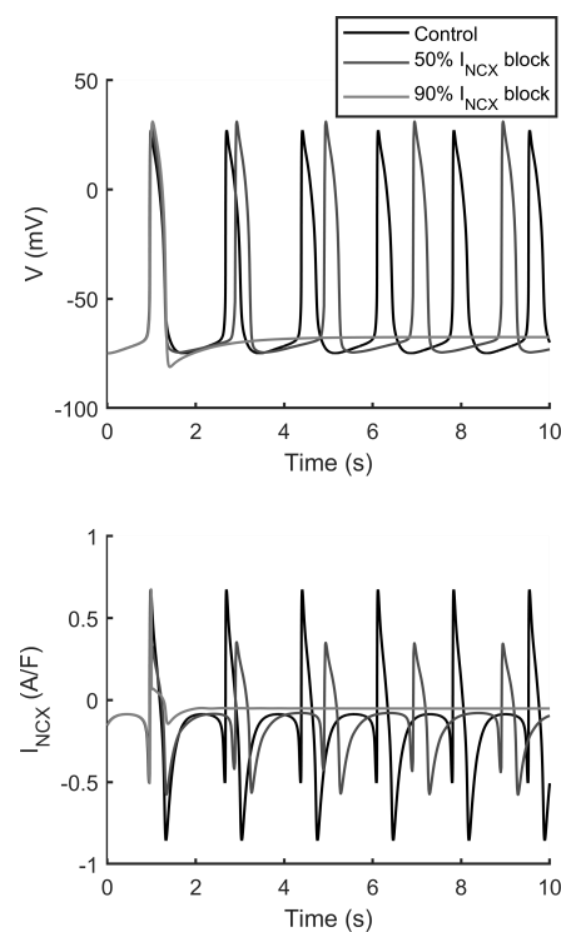

Figure 2. Effect of increasing $\mathrm{I}_{\mathrm{NCX}}$ block on the Paci2019 model.
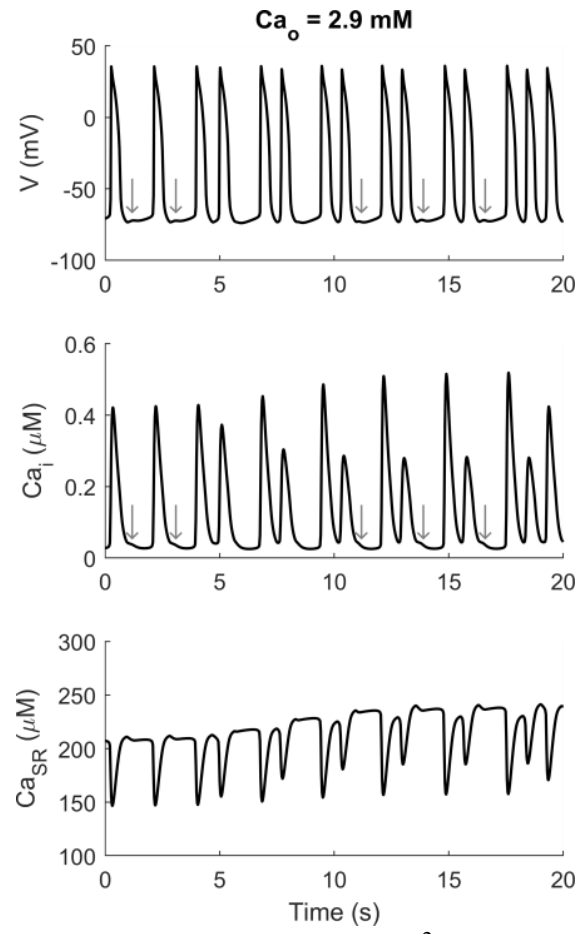

Figure 3. DADs as consequence of $\mathrm{Ca}^{2+}$ overload due to increased extracellular $\mathrm{Ca}^{2+}$ concentration.

we presented an updated hiPSC-CM model that summarizes both the mechanisms for the AP automaticity and that can be used to attempt to verify these hypotheses. 

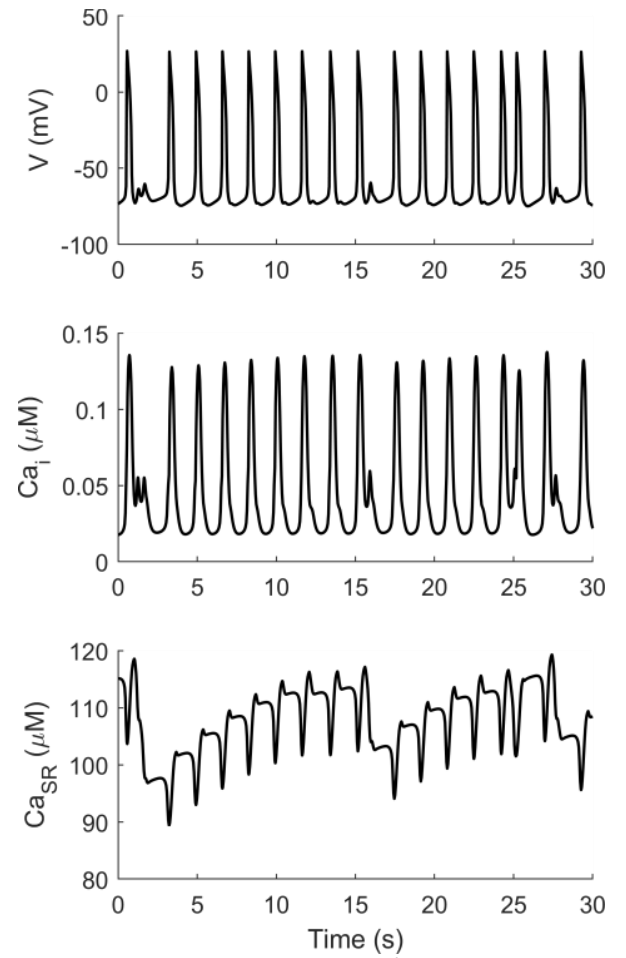

Figure 4. DADs due to abnormal $\mathrm{Ca}^{2+}$ handling.
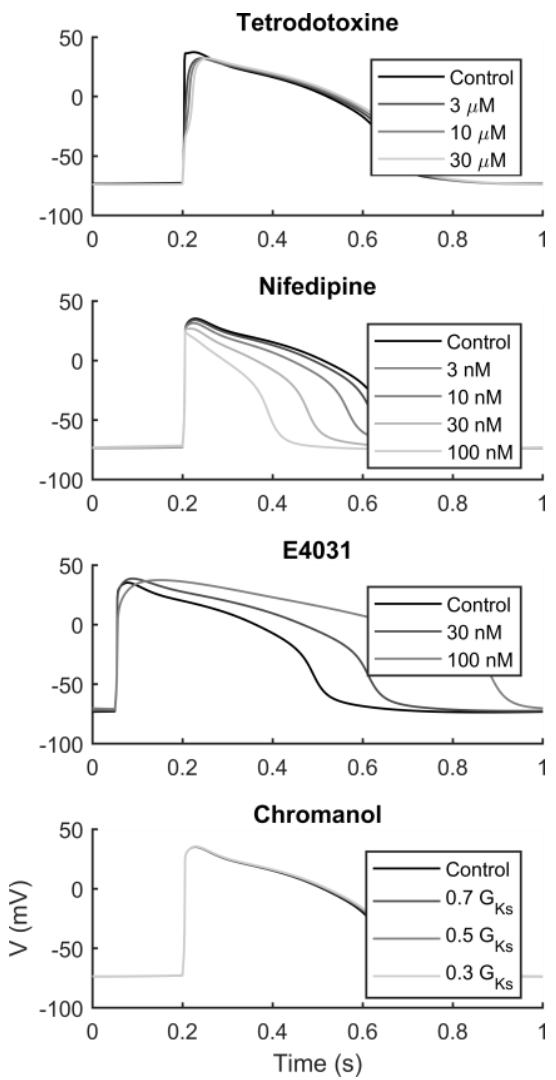

Figure 5. Model responses to current blockers.

\section{Acknowledgments}

Dr. Michelangelo Paci was supported by the Academy of Finland (decision number 307967).

\section{References}

[1] Paci $\mathrm{M}$ et al. Human induced pluripotent stem cell-derived versus adult cardiomyocytes: an in silico electrophysiological study on effects of ionic current block. Br J Pharmacol. 2015;172(21):5147-60.

[2] Paci M et al. Phenotypic variability in LQT3 human induced pluripotent stem cell-derived cardiomyocytes and their response to antiarrhythmic pharmacologic therapy: An in silico approach. Hear Rhythm. 2017;14(11):1704-12.

[3] Paci M et al. Automatic optimization of an in silico model of human iPSC derived cardiomyocytes recapitulating calcium handling abnormalities. Front Physiol. 2018;9(June):709.

[4] Koivumäki JT et al. Structural Immaturity of Human iPSCDerived Cardiomyocytes: In Silico Investigation of Effects on Function and Disease Modeling. Front Physiol. 2018;9(February):1-17.

[5] Paci M et al. A population of in silico models to face the variability of human induced pluripotent stem cell-derived cardiomyocytes: the hERG block case study. In: Computing in Cardiology. 2016. p. 1189-92.

[6] Paci M et al. Computational models of ventricular- and atriallike human induced pluripotent stem cell derived cardiomyocytes. Ann Biomed Eng. 2013;41(11):2334-48.

[7] Chauveau S et al. Induced Pluripotent Stem Cell-Derived Cardiomyocytes Provide In Vivo Biological Pacemaker Function. Circ Arrhythmia Electrophysiol. 2017;10(5):1-10.

[8] Kim JJ et al. Mechanism of automaticity in cardiomyocytes derived from human induced pluripotent stem cells. J Mol Cell Cardiol. 2015;81:81-93.

[9] Ma J et al. High purity human-induced pluripotent stem cellderived cardiomyocytes: electrophysiological properties of action potentials and ionic currents. AJP - Hear Circ Physiol. 2011;301(5):H2006-17.

[10]Sartiani L et al. Developmental changes in cardiomyocytes differentiated from human embryonic stem cells: a molecular and electrophysiological approach. Stem Cells. 2007;25(5):1136-44.

[11]Rast $G$ et al. Influence of field potential duration on spontaneous beating rate of human induced pluripotent stem cell-derived cardiomyocytes: Implications for data analysis and test system selection. J Pharmacol Toxicol Methods. 2016;82:74-82.

[12]Volders PG et al. Progress in the understanding of cardiac early afterdepolarizations and torsades de pointes: time to revise current concepts. Cardiovasc Res. 2000;46(3):376-92.

Address for correspondence:

Michelangelo Paci.

Tampere University,

Arvo Ylpön katu 34, D 219, FI-33520, Tampere, Finland.

michelangelo.paci@tuni.fi 